\title{
ON THE EXTENSION OF BOUNDARY INTEGRABLE ALMOST COMPLEX STRUCTURE ${ }^{1}$
}

\author{
BY GARO K. KIREMIDJIAN
}

Communicated by I. M. Singer, March 6, 1974

1. Introduction. Let $\left\{M, M^{\prime}\right\}$ be a finite Kähler manifold, i.e., $M^{\prime}$ is a complex Kähler manifold, $M$ is an open submanifold of $M^{\prime}$ with compact closure, $M_{0}=b M$, the boundary of $M$, is a $C^{\infty}$ submanifold of $M^{\prime}$, and for each $p \in M_{0}$ there exists a coordinate neighborhood $U$ of $p$ with real coordinates $t^{1}, \cdots, t^{2 n-1}, r$ such that $r(q)<0$ for $q \in U \cap M$ and $r(q)>0$ for $q \in U \cap\left(M^{\prime}-M\right)$. It is assumed that the following conditions hold:

A. For each boundary point the Levi form has at least two positive eigenvalues.

B. There exists a constant $c_{0}>0$ such that for all $u \in C^{0, q}(\bar{M}, \Theta)$, $q=1,2 \quad((2 \square-\triangle) u, u) \geqq c_{0}(u, u)$ where $\Theta$ is the holomorphic tangent bundle of $M^{\prime}, C^{p, q}(\bar{M}, \Theta)$ is the space of all $C^{\infty} \Theta$-valued $(p, q)$-forms extendible to a neighborhood of $\bar{M}, \square$ (resp., $\triangle$ ) is the complex (resp., the real) Laplacian on $C^{p, q}(\bar{M}, \Theta)$ and $(, \quad)$ is the $L_{2}$-inner product over $M$ (see [2]).

Then the main result of this note states that a sufficiently small integrable almost-complex structure on $M_{0}$ can be extended to a complex structure on $M$. A complete proof will appear elsewhere; a brief outline follows.

However, we first take a closer look at condition B. Let $D$ be the covariant differentiation operator associated with the connection $\theta$ of the metric $g$ on $M^{\prime}$, i.e.,

$$
D u=d u+\theta \wedge u=\delta u+\tilde{\partial} u
$$

for $u \in C^{p, q}(\bar{M}, \Theta)$. Let $D^{*}$ and $\delta^{*}$ be the formal adjoints of $D$ and $\delta$, respectively. Then $\triangle=D D^{*}+D^{*} D$ and $\square=\bar{\partial} \bar{\partial}^{*}+\bar{\partial}^{*} \bar{\partial}$. Since $g$ is Kähler, $\triangle=2 \square-K, K=\sqrt{ }-1(e(s) \Lambda-\Lambda e(s))$, where

$$
e(s) u=\bar{\partial} \theta \wedge u, \quad \Lambda u=*^{-1}(\rho \wedge * u),
$$

* is the Hodge star operator and $\rho$ is the Kähler form of $g$. We refer

AMS (MOS) subject classifications (1970). Primary 32G99; Secondary 32G05, $35 \mathrm{~J} 60,32 \mathrm{~J} 25$.

${ }^{1}$ This research was supported in part by NSF grant GP $33942 X 1$. 
to [3, pp. 482-483], for verification of this identity. Hence, condition B requires the existence of a constant $c_{0}>0$ such that $(K u, u) \geqq c_{0}(u, u)$ for all $u \in C^{0, q}(\bar{M}, \Theta), q=1,2$. Now it is established in [2, p. 276], that if the scalar curvature is sufficiently negative, then one has the stronger result $\langle K u, u\rangle_{x} \geqq c_{0}\langle u, u\rangle_{x}$ for all $x \in M^{\prime}$, where $\langle,\rangle_{x}$ is the inner product at the point $x$, i.e., $\Theta$ is $W^{0, q}$-elliptic. It is also shown in [2] that the criterion of $W$-ellipticity is satisfied for a large class of bounded homogeneous domains in $\boldsymbol{C}^{n}$ provided with the Bergman metric. More generally, let $M^{\prime}$ be a manifold whose universal covering space $\tilde{M}^{\prime}$ is isomorphic to $D_{1} \times \cdots \times D_{r}$, where $D_{i}$ is a bounded irreducible symmetric domain with $\operatorname{dim}_{C} D_{i} \geqq 3$. Then $\Theta$ is $W^{0, q}$-elliptic for $0 \leqq q \leqq 2$, and condition B will hold for any relatively compact open submanifold $M$ of $M^{\prime}$ with smooth boundary.

2. Definitions and notation. Let $M_{0}$ be a $C^{\infty}$ manifold of real dimension $2 n-1$ and let $C T M_{0}$ be the complexified tangent bundle.

2.1. Definition. An almost-complex structure on $M_{0}$ is given by a complex subbundle $E^{\prime \prime}$ of $C T M_{0}$ of fiber complex dimension $n-1$ such that $E^{\prime \prime} \cap \bar{E}^{\prime \prime}=\{0\}$.

2.2. Definition. The almost-complex structure $E^{\prime \prime}$ on $M_{0}$ is integrable if, for any two sections $L$ and $L^{\prime}$ of $E^{\prime \prime}$ over an open set $U$ of $M_{0},\left[L, L^{\prime}\right]$ is also a section of $E^{\prime \prime}$.

We now assume that $M_{0}$ is the boundary of a finite complex manifold $\left\{M, M^{\prime}\right\}$. The complex structure on $M^{\prime}$ induces an integrable almostcomplex structure $T^{\prime \prime}$ on $M_{0}$.

2.3. Definition. The almost-complex structure $E^{\prime \prime}$ on $M_{0}$ is of finite distance from $T^{\prime \prime}$ if $\pi^{\prime \prime} \mid E^{\prime \prime}: E^{\prime \prime} \rightarrow T^{\prime \prime}$ is an isomorphism where $\pi^{\prime \prime}: C T M_{0} \rightarrow T^{\prime \prime}$ is the projection.

In this case $E^{\prime \prime}=\left\{X-\tau \circ \varphi(X) \mid X \in T^{\prime \prime}\right\}$ where $\tau: \Theta \mid M_{0} \rightarrow T^{\prime} \oplus C F$ is an isomorphism, $T^{\prime}=\bar{T}^{\prime \prime}, C F$ is the complexification of a real one-dimensional subbundle $F$ of $T M_{0}$ such that $C T M_{0}=T^{\prime} \oplus T^{\prime \prime} \oplus C F$ and

$$
\varphi=-\tau^{-1} \circ\left(\mathrm{id}-\pi^{\prime \prime}\right) \circ\left(\pi^{\prime \prime} E^{\prime \prime}\right)^{-1}: T^{\prime \prime} \rightarrow \Theta \mid M_{0},
$$

i.e., $\varphi$ is a $\Theta \mid M_{0}$-valued $C^{\infty}$ differential form on $M_{0}$ of type $(0,1)_{b}$. Conversely, any such differential form $\varphi$ gives rise to an almost complex structure $E^{\prime \prime}$ on $M_{0}$. We will denote $E^{\prime \prime}$ by $T_{\varphi}^{\prime \prime}$. As in the case of complex manifolds, there exists a $\Theta \mid M_{0}$-valued $C^{\infty}$ differential form $\Phi$ on $M_{0}$ of type $(0,2)_{b}$ such that $\Phi=0$ if and only if $T_{\varphi}^{\prime \prime}$ is integrable.

Let $\varphi$ be a $T^{\prime}$-valued form and let $\omega \in C^{0,1}(\bar{M}, \Theta)$ be such that $t \omega$, the complex tangential part of $\omega$, is equal to $\varphi$ on $M_{0}$. Let $\Omega=\bar{\partial} \omega-[\omega, \omega]$. If $T_{\omega}^{\prime \prime}$ is the almost complex structure on $M$ induced by $\omega$, then one can show that $T_{\varphi}^{\prime \prime}=C T M_{0} \cap T_{\omega}^{\prime \prime}$ and $t \Omega=0$ on $M_{0}$ if and only if $\Phi=0$. 
3. The main result. Now we can formulate the following extension problem.

THEOREM. Let $\left\{M, M^{\prime}\right\}$ be a finite complex Kähler manifold such that conditions $\mathrm{A}$ and $\mathrm{B}$ in $\S 1$ are satisfied. Let $\varphi$ be a $T^{\prime}$-valued $C^{\infty}$ differential form of type $(0,1)_{b}$ with sufficiently small Hölder norm $|\varphi|_{k+\alpha}, 0<\alpha<1$, for some integer $k>0$ depending on $n$. Assume that $T_{\varphi}^{\prime \prime}$ is integrable. Then there exists $\omega \in C^{0,1}(\bar{M}, \Theta)$ such that $\Omega=0$ and $t \omega=\varphi$ on $M_{0}$.

We first consider the quadratic form

$$
Q(u, v)=\frac{1}{2}\left[(D u, D v)+\left(D^{*} u, D^{*} v\right)+(K u, v)\right]-2([\psi, u], \bar{\partial} v)
$$

for some $\psi \in C^{0,1}(\bar{M}, \Theta)$ with sufficiently small norm and $u, v \in \mathfrak{B}=$ $\left\{\omega \in C^{0,1}(\bar{M}, \Theta) \mid t \omega=0\right.$ on $\left.M_{0}\right\}$. One can easily check that by condition B, const $N^{2}(u) \leqq|\operatorname{Re} Q(u, u)| \leqq$ const $N^{2}(u)$ where $\operatorname{Re}$ stands for the real part of $Q(u, u)$, and $N^{2}(u)=\|u\|^{2}+\|D u\|^{2}+\left\|D^{*} u\right\|^{2}$. Hence, if $\|u\|_{s}$ is the Sobolev $s$-norm of $u$, then $\|u\|_{1} \leqq$ const $|\operatorname{Re} Q(u, u)|$.

It follows from the theory developed in [1] and [4] that for each $\sigma \in C^{0,1}(\bar{M}, \Theta)$ there exists a unique $u \in \mathfrak{B}$ such that $Q(u, v)=(\sigma, v)$ for all $v \in \hat{\mathfrak{B}}$, the completion of $\mathfrak{B}$ with respect to the norm $N$ such that

$$
\begin{gathered}
\|u\|_{s+2} \leqq c_{s}\|\sigma\|_{s} \\
L_{\psi} u=\frac{1}{2}\left(D D^{*}+D^{*} D+K\right) u-2 \bar{\partial}^{*}[\psi, u]=\sigma \\
t D^{*} u=0 \text { on } M_{0} \\
|u|_{k+\alpha+2} \leqq c_{k}^{\prime}|f|_{k+\alpha}
\end{gathered}
$$

for sufficiently large $k$. The constants $c_{s}$ and $c_{k}^{\prime}$ depend on $s$ and $k$ and on the derivatives of $\psi$ up to order $s$ and $k$, respectively. If $|\psi|_{k+\alpha}$ is sufficiently small we may assume that $c_{k}^{\prime}$ in (4) depends only on $k$.

We observe that $D^{*} u=-* \bar{\partial} * u-* \tilde{\partial} * u$, and since $u$ is a form of type $(0,1), \bar{\partial} * u=0$ and $D^{*} u=\bar{\partial}^{*} u$. On the other hand for a Kähler metric $g$ the complex Laplacian $\square=\bar{\partial} \bar{\partial}^{*}+\bar{\partial}^{*} \bar{\partial}$ is $\frac{1}{2}\left(D D^{*}+D^{*} D+K\right)$, and if $\sigma=\bar{\partial}^{*} h$ for $h \in C^{0,2}(\bar{M}, \Theta)$, then (3) and Stokes' theorem imply that $L_{\psi} u=\sigma$ if and only if

$$
\bar{\partial}^{*} \bar{\partial} u-2 \bar{\partial}^{*}[\psi, u]=\bar{\partial}^{*} h .
$$

We now consider the nonlinear differential system $\bar{\partial}^{*} \Omega=0$. Let $\omega_{0} \in$ $C^{\mathbf{0}, \mathbf{1}}(\bar{M}, \Theta)$ be an extension of $\varphi$ such that $\left|\omega_{0}\right|_{k+\alpha} \leqq$ const $|\varphi|_{k+\alpha}$. One can inductively construct a sequence of approximate solutions $\omega_{m+1}=\omega_{m}+u_{m}$, where $u_{m}$ is the solution of (5) with $t u_{m}=t \bar{\partial}^{*} u_{m}=0$ on $M_{0}, \psi=\omega_{m}$, $h=-\Omega_{m}=-\bar{\partial} \omega_{m}+\left[\omega_{m}, \omega_{m}\right]$. Since $\left|\bar{\partial} * \Omega_{m}\right|_{k+\alpha-2} \leqq$ const $\left|u_{m}\right|_{k+\alpha},(4)$ implies that there exists a constant $c>0$ such that $\left|\omega_{m+1}-\omega_{m}\right|_{k+\alpha} \leqq c\left|\omega_{m}-\omega_{m-1}\right|_{k+\alpha}^{2}$ 
for $m=1,2, \cdots$. This is enough to conclude that there exists a $\Theta$-valued form $\omega$ of type $(0,1)$ and of class $C^{k+\alpha}$ on $\bar{M}$ such that $\bar{\partial}^{*} \Omega=0, t \omega=\varphi$ on $M_{0}$, and $|\omega|_{k+\alpha} \leqq$ const $|\varphi|_{k+\alpha}$.

Now it can easily be shown that $\bar{\partial} \Omega=2[\omega, \Omega]$. By condition $A$ and the fact that the normal part of $* \# \Omega$ vanishes on $M_{0}$, the basic estimate of the $\bar{\partial}$-Neumann problem holds for $* \# \Omega$, i.e.,

$$
E(* \# \Omega) \leqq \operatorname{const}\left(\|\Omega\|^{2}+\|\bar{\partial} \Omega\|^{2}+\left\|\bar{\partial}^{*} \Omega\right\|^{2}\right) .
$$

For the definition of the norm $E$, we refer to [5] and [6]; the operators * and \# are defined in [2]. Then by condition $B$ and the complete continuity of $E$, one can obtain the estimate $\|\bar{\partial} \Omega\| \leqq c_{0}|\omega|_{1, \alpha}\|\bar{\partial} \Omega\|$ for some constant $c_{0}$. Thus $\bar{\partial} \Omega=0$ if $|\varphi|_{k+\alpha}$ is sufficiently small. Since $t \Omega=0$ on $M_{0}, \bar{\partial} \Omega=0$, and $\bar{\partial}^{*} \Omega=0$, condition $B$ implies that $\Omega=0$.

Finally, it follows from the construction of approximate solutions that $\omega=\omega_{0}+w$, where $w$ is of class $C^{k+\alpha}$ and $\bar{\partial}^{*} w=0$. Then $\bar{\partial}^{*}(\bar{\partial} \omega-[\omega, \omega])=0$ can be expressed as

$$
\square w-\bar{\partial}^{*}\left(2\left[\omega_{0}, w\right]+[w, w]\right)=\delta^{*}\left(\left[\omega_{0}, \omega_{0}\right]-\delta \omega_{0}\right) .
$$

This equation is elliptic if $|\varphi|_{k+\alpha}$ is sufficiently small. Since $\omega_{0}$ is of class $C^{\infty}, w$ is also of class $C^{\infty}$.

\section{REFERENCES}

1. S. Agmon, A. Douglis and L. Nirenberg, Estimates near the boundary for solutions of elliptic partial differential equations satisfying general boundary conditions. II, Comm. Pure Appl. Math. 17 (1964), 35-92. MR 28 \#5252.

2. A. Andreotti and E. Vesentini, On deformations of discontinuous groups, Acta Math. 112 (1964), 249-298. MR 29 \#6503.

3. E. Calabi and E. Vesentini, On compact, locally symmetric Kähler manifolds, Ann. of Math. (2) 71 (1960), 472-507. MR 22 \#1922b.

4. G. Fichera, Linear elliptic differential systems and eigenvalue problems, Lecture Notes in Math., no. 8, Springer-Verlag, Berlin and New York, 1965. MR 35 \#536.

5. J. J. Kohn, Harmonic integrals on strongly pseudo-convex manifolds. I, Ann. of Math. (2) 78 (1963), 112-148. MR 27 \#2999.

6. J. J. Kohn and $\mathrm{H}$. Rossi, On the extension of holomorphic functions from the boundary of a complex manifold, Ann. of Math. (2) 81 (1965), 451-472. MR 31 \#1399.

Department of Mathematics, Stanford University, Stanford, California 94305 